\title{
Elevated fibrinogen and fibrin degradation product are associated with poor outcome in COVID-19 patients: A meta-analysis
}

Johanes Nugroho $^{\mathrm{a}, \mathrm{c}, *}$, Ardyan Wardhana $^{\mathrm{b}}$, Eka Prasetya Mulia $^{\mathrm{c}}$, Irma Maghfirah $^{\mathrm{c}}$, Dita Aulia Rachmi ${ }^{\mathrm{c}}$, Maya Qurota $\mathrm{A}^{\prime} \mathrm{yun}^{\mathrm{c}}$ and Imanita Septianda ${ }^{\mathrm{c}}$

${ }^{a}$ Department of Cardiology and Vascular Medicine, Faculty of Medicine, Universitas Airlangga, Surabaya, Indonesia

${ }^{\mathrm{b}}$ Faculty of Medicine, Universitas Surabaya, Indonesia

${ }^{\mathrm{c}}$ Dr. Soetomo General Hospital, Surabaya, Indonesia

\begin{abstract}
.
INTRODUCTION: COVID-19 is a systemic infection with a significant impact on coagulation which manifests in thromboembolism. There is an unknown relationship of which coagulation profile parameter at presentation has an association with poor outcome in COVID-19.

OBJECTIVE: This meta-analysis aimed to determine the relationship between fibrinogen and FDP with poor outcome in COVID-19 patients.

METHODS: A systematic search of all observational studies or trials involving adult patients with COVID-19 that had any data fibrinogen or FDP on admission was carried out using the PubMed, Science Direct, Scopus, ProQuest, and MedRxiv databases. We assessed the methodological quality assessment using the NIH Quality Assessment Tool. We performed random-effects inverse-variance weighting analysis using mean difference (MD).

RESULTS: A total of 17 studies (1,654 patients) were included in this meta-analysis. It revealed a higher mean of fibrinogen levels on admission in patients with severe case compared to those with non-severe case $(\mathrm{MD}=0.69$, [95\% CI: 0.44 to 0.94$]$, $p<0.05 ; \mathrm{I} 2=72 \%, p<0.05)$. Non-survivor group had a pooled higher mean difference of fibrinogen values on admission $(\mathrm{MD}=0.48$ [95\% CI: 0.13 to 0.83 ] $p<0.05 ; \mathrm{I} 2=38 \%, p=0.18)$. Higher FDP on admission was found in poor outcome (composite of severity, critically ill, and mortality) compared to good outcome (4 studies, MD = 4.84 [95\% CI: 0.75 to 8.93 ], $p<0.05 ; \mathrm{I} 2=86 \%, p<0.05)$.

CONCLUSION: Elevated fibrinogen and FDP level on admission were associated with an increase risk of poor outcome in COVID-19 patients.
\end{abstract}

Keywords: COVID-19, fibrinogen, fibrin degradation product, severity, mortality, prognostic factor

\section{Introduction}

COVID-19 is a systemic infection with a significant impact on coagulation which manifests in thromboembolism. Furthermore, disseminated intravascular coagulopathy appeared in most of the deaths [1]. At the late stages of COVID-19, a common coagulation activation and secondary hyperfibrinolysis condition occurred and accompanied with elevated levels of fibrin-related markers (D-dimer and fibrin degradation product [FDP]) and low fibrinogen level [2].

At initial presentation of coagulopathy, patients with COVID-19 presents with a prominent elevation of fibrinogen and FDP [3]. Some studies showed that significant coagulopathy was present in

*Corresponding author: Johanes Nugroho. E-mail: j.nugroho.eko@fk.unair.ac.id. 
COVID-19 patients with elevated fibrinogen at presentation [4]. Nevertheless, there is an unknown relationship of which coagulation profile parameter at presentation has an association with severity and mortality in COVID-19. This meta-analysis aimed to determine the relationship between fibrinogen and FDP with poor outcome in COVID-19 patients.

\section{Methods}

This study in accordance with the PRISMA guidelines (Preferred Reporting Items for Systematic Reviews and Meta-Analyses) [5]. We selected all observational studies involving adult patients with COVID-19 that had any fibrinogen and FDP data for comparison severe vs. non-severe, and survivor vs. non-survivor. We excluded any study that had missing required data, fibrinogen and FDP data not collected on admission, and data not presented in numerical variables.

A systematic search of the literature was carried out on May 15, 2020, after receiving approval from the Institutional Review Board. Five different databases (PubMed, Science Direct, Scopus, ProQuest, and MedRxiv) were used to perform a systematic search of all the literature using the keywords "intensive" and "laboratory" and "fibrinogen" OR "fibrinogen degradation products OR FDP" and "COVID-19" or "coronavirus 2019" or "2019-nCoV" or "SARS-CoV-2" in the title, abstract, and medical subject heading (MeSH). Reference lists of the included studies were also screened to identify additional relevant studies.

Three investigators independently screened and assessed titles and abstracts before full-text retrieval. The full papers that potentially met the inclusion/exclusion criteria were reviewed by the two authors for final inclusion. Subsequently, two investigators extracted the data, including authors, year of publication, location, study design, peer-reviewed publication status, severity measurement, and fibrinogen and FDP level in each comparison group. All extracted data were recorded with a dedicated form on an Excel spreadsheet.

The primary outcome in our meta-analysis was fibrinogen on admission based on severity. All definition of severity was used in this meta-analysis. If the study categorized severity into 3 or 4 groups, we combined the data between mild and moderate groups into one group as non-severe; severe and critical group into one group as severe. The average of their mean and standard deviation was calculated using the formula in Table 7.7.a of the Cochrane Handbook [6]. The secondary outcomes were fibrinogen on admission based on mortality and FDP level on admission based on composite poor outcome that comprised of severity and mortality.

Two authors independently assessed the methodological quality assessment using the NIH Quality Assessment Tool for Observational Cohort and Cross-Sectional Studies [7]. Publication bias assessed using visual inspection of funnel plots and Egger's regression test [8]. We conducted the meta-analysis using mean difference (MD) for fibrinogen and FDP values. Mean and standard deviation were extrapolated from sample size, median, and interquartile range (IQR), according to Wan et al. [9]. We employed a random-effects and inverse-variance weighting using Review Manager (RevMan v5.3 2014). We performed subgroup analysis based on study design. Sensitivity analysis was done using leave-one-out method to assess the cause of heterogeneity. We evaluated between and within-study heterogeneity using the $\mathrm{I}^{2}$ statistic.

\section{Results}

Initial search provided 161 records from the PUBMED, 100 records from Science Direct, 107 records from ProQuest, 82 records from Scopus database, 111 records from Medxriv database, and 


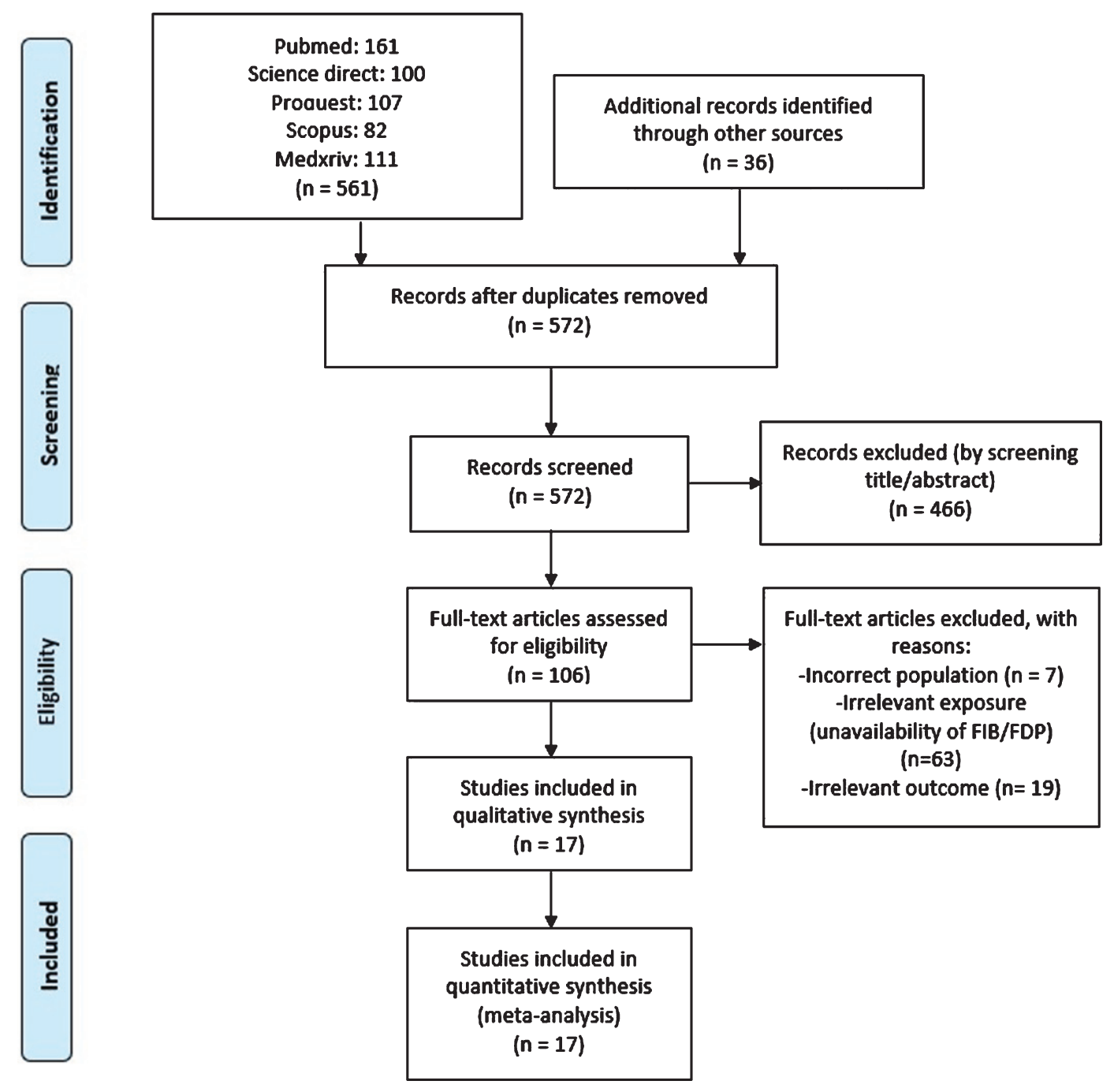

Fig. 1. Study flow chart (as per PRISMA guideline).

36 records from other sources as shown in Fig. 1. After removing the duplicate and screening of title and abstracts, 106 full texts were then assessed for eligibility. A total of 89 were excluded because of incorrect patient population $(n=7)$, unavailability of data on fibrinogen or fibrin degradation product $(n=63)$, and irrelevant outcome $(n=19)$. Totally, we included 17 studies $(1,654$ patients $)$ for analysis.

The baseline characteristics of the included studies are presented in Table 1. Fifteen studies were retrospective, and two studies were prospective observational. Ten studies have already been undergone peer-review [2, 10-18]. Most of the studies classified the severity according to the National Health Commission of People's Republic of China.

We assessed all studies' quality based on NHLBI quality assessment resulting in good and fair methodology qualities in all included studies (Table 1). None of the studies was considered to be seriously flawed. The analyses were rigorous, and the conclusions drawn by the studies were credible. However, most studies did not assess exposure prior to outcome measurement and might not have sufficient timeframe for outcomes to occur due to their cross-sectional design. 
Table 1

Baseline characteristics of included studies

\begin{tabular}{|c|c|c|c|c|c|c|c|c|c|c|c|c|c|c|c|c|c|}
\hline No & o Author & $\begin{array}{l}\text { Study } \\
\text { design }\end{array}$ & Hospital & $\begin{array}{l}\text { Town, } \\
\text { Country }\end{array}$ & Period & Samples (n) & Male (\%) & Age, y & $\begin{array}{l}\text { Age } \\
\text { difference }\end{array}$ & $\begin{array}{l}\text { Outcome /No. of } \\
\text { group }\end{array}$ & $\begin{array}{l}\text { FIB }(g / L) \\
\text { Mean } \pm S D\end{array}$ & $\begin{array}{l}\text { FDP }(\mu \mathrm{g} / \mathrm{ml}) \\
\text { Mean } \pm \mathrm{SD}\end{array}$ & $\begin{array}{l}\text { Time of } \\
\text { diagnosis }\end{array}$ & HTN $(\%)$ & CVD (\%) & DM (\%) & $\begin{array}{l}\text { Study } \\
\text { quality }\end{array}$ \\
\hline 1 & $\begin{array}{l}\text { Zhang H, } \\
2020[20]\end{array}$ & Retros. & $\begin{array}{l}\text { Chongqing Public } \\
\text { Health Medical } \\
\text { Center }\end{array}$ & $\begin{array}{l}\text { Chongqing, } \\
\text { China }\end{array}$ & $\begin{array}{l}\text { Feb 11-28, } \\
2020\end{array}$ & 43 (14 vs 29$)$ & 35.7 vs 58.6 & $\begin{array}{l}61.7 \pm 9.22 \\
\text { vs } \\
44.34 \pm 15.84\end{array}$ & $p<0.001$ & $\begin{array}{l}\text { Severity } / 2 \text { based } \\
\text { on NHC China }\end{array}$ & $\begin{array}{l}4.70 \pm 1.98 \\
\text { vs } \\
3.50 \pm 1.35\end{array}$ & $\mathrm{n} / \mathrm{a}$ & $\begin{array}{l}\text { On } \\
\text { admission }\end{array}$ & 7.1 vs 10.3 & $\mathrm{n} / \mathrm{a}$ & 21.4 vs 10.3 & Good \\
\hline 2 & $\begin{array}{l}\text { Qian GQ, } \\
2020[11]\end{array}$ & Retros. & $\begin{array}{l}\text { Five hospitals in } \\
\text { Zhejiang province }\end{array}$ & $\begin{array}{l}\text { Zhejiang } \\
\text { province, } \\
\text { China }\end{array}$ & $\begin{array}{l}\text { Jan 20-Feb } \\
11,2020\end{array}$ & 91 (9 vs 82 ) & $\mathrm{n} / \mathrm{a}$ & $\begin{array}{l}66(54-80) \\
\text { vs } 49 \\
(35.3-56)\end{array}$ & $p<0.001$ & $\begin{array}{l}\text { Severity } / 2 \text { based } \\
\text { on NHC China }\end{array}$ & $\begin{array}{l}3.89 \pm 0.38 \\
\text { vs } \\
3.36 \pm 1.09\end{array}$ & $\mathrm{n} / \mathrm{a}$ & $\begin{array}{l}\text { On } \\
\text { admission }\end{array}$ & $\mathrm{n} / \mathrm{a}$ & n/a & $\mathrm{n} / \mathrm{a}$ & Good \\
\hline 3 & $\begin{array}{l}\text { Chen X, } \\
2020[21]\end{array}$ & Retros. & $\begin{array}{l}\text { Hospital of } \\
\text { Changsha and } \\
\text { Loudi Central } \\
\text { Hospital }\end{array}$ & $\begin{array}{l}\text { Hunan, } \\
\text { China }\end{array}$ & $\begin{array}{l}\text { Jan 23-Feb } \\
14,2020\end{array}$ & 58 (12 vs 46$)$ & 54 vs 48.9 & $\begin{array}{l}58.83 \pm 18.31 \\
\text { vs } \\
42.46 \pm 15.55\end{array}$ & $p=0.001$ & $\begin{array}{l}\text { Severity/3 based } \\
\text { on NHC China }\end{array}$ & $\begin{array}{l}4.59 \pm 1.07 \\
\text { vs } \\
4.37 \pm 1.11\end{array}$ & $\mathrm{n} / \mathrm{a}$ & $\begin{array}{l}\text { On } \\
\text { admission }\end{array}$ & 38 vs 8.3 & 7 vs 2.9 & 14 vs 6.2 & Fair \\
\hline 4 & $\begin{array}{l}\text { Zhu Z, } 2020 \\
{[12]}\end{array}$ & Retros. & Hwa Mei Hospital & $\begin{array}{l}\text { Zhejiang } \\
\text { province, } \\
\text { China }\end{array}$ & $\begin{array}{l}\text { Jan 23-Feb } \\
20,2020\end{array}$ & $\begin{array}{l}127 \text { (16 vs } \\
111)\end{array}$ & $\begin{array}{l}56.25 \text { vs } \\
65.77\end{array}$ & $\begin{array}{l}57.50 \pm 11.70 \\
\text { vs } \\
49.95 \pm 15.52\end{array}$ & $p=0.03$ & $\begin{array}{l}\text { Severity } / 2 \text { based } \\
\text { on NHC China }\end{array}$ & $\begin{array}{l}5.49 \pm 2.14 \\
\text { vs } 4.45 \pm 1.4\end{array}$ & $\mathrm{n} / \mathrm{a}$ & $\mathrm{n} / \mathrm{a}$ & 50 vs 20.72 & 12.5 vs 3.6 & 0 vs 9.01 & Good \\
\hline 5 & $\begin{array}{l}\text { Giamarellos- } \\
\text { Bourboulis } \\
\text { E, } 2020[13]\end{array}$ & Pros & $\begin{array}{l}\text { Sotiria Athens } \\
\text { General Hospital } \\
\text { and Attikon } \\
\text { University General } \\
\text { Hospital }\end{array}$ & $\begin{array}{l}\text { Athens, } \\
\text { Greece }\end{array}$ & $\begin{array}{l}\text { March 3-30, } \\
2020\end{array}$ & 54 (28 vs 26$)$ & 89.3 vs 57.5 & $\begin{array}{l}67.8 \pm 10.8 \\
\text { vs } \\
59.2 \pm 10.3\end{array}$ & $p<0.001$ & $\begin{array}{l}\text { Severity(severe } \\
\text { respiratory } \\
\text { failure)/2 }\end{array}$ & $\begin{array}{l}6.94 \pm 1.89 \\
\text { vs } \\
5.29 \pm 1.53\end{array}$ & $\mathrm{n} / \mathrm{a}$ & $\mathrm{n} / \mathrm{a}$ & $\mathrm{n} / \mathrm{a}$ & $\begin{array}{l}17.9 \text { vs } 7.7 \\
\text { (CAD) }\end{array}$ & 21.4 vs 15.4 & Fair \\
\hline 6 & $\begin{array}{l}\text { Zheng C, } \\
2020[14]\end{array}$ & Retros. & $\begin{array}{l}\text { Wuhan Union } \\
\text { Hospital }\end{array}$ & $\begin{array}{l}\text { Wuhan, } \\
\text { China }\end{array}$ & Feb 15,2020 & 55 (21 vs 34$)$ & 38.1 vs 47.1 & $\begin{array}{l}\text { 62(29-91) vs } \\
59(29-77)\end{array}$ & $p=0.37$ & Severity/2 & $\begin{array}{l}5.5 \pm 1.27 \mathrm{vs} \\
3.73 \pm 0.79\end{array}$ & $\begin{array}{l}6.25 \pm 4.55 \\
\text { vs } \\
4.73 \pm 3.39\end{array}$ & $\mathrm{n} / \mathrm{a}$ & $\mathrm{n} / \mathrm{a}$ & $\mathrm{n} / \mathrm{a}$ & $\mathrm{n} / \mathrm{a}$ & Good \\
\hline 7 & $\begin{array}{l}\text { Sun Y, } 2020 \\
{[15]}\end{array}$ & Retros. & $\begin{array}{l}\text { Chinese PLA } \\
\text { General Hospital }\end{array}$ & $\begin{array}{l}\text { Beijing, } \\
\text { China }\end{array}$ & n/a & 63 (19 vs 44$)$ & 52.8 vs 70.15 & $\begin{array}{l}42.25 \pm 17.49 \\
\text { vs } \\
59.36 \pm 15.58\end{array}$ & $p=0.000$ & $\begin{array}{l}\text { Severity/4 based } \\
\text { on NHC China }\end{array}$ & $\begin{array}{l}3.66 \pm 1.76 \\
\text { vs } \\
2.94 \pm 1.01\end{array}$ & $\mathrm{n} / \mathrm{a}$ & $\begin{array}{l}\text { On } \\
\text { admission }\end{array}$ & 21.1 vs 18.2 & $\begin{array}{l}10.5 \text { vs } 0 \\
\text { (cardiac } \\
\text { arrhythmia) }\end{array}$ & 15.8 vs 4.5 & Good \\
\hline 8 & $\begin{array}{l}\text { Zeng L, 2020 } \\
{[4,5,22]}\end{array}$ & Retros. & $\begin{array}{l}\text { Third People's } \\
\text { Hospital of } \\
\text { Shenzhen }\end{array}$ & $\begin{array}{l}\text { Shenzhen, } \\
\text { China }\end{array}$ & $\begin{array}{l}\text { Jan 11-Feb } \\
29,2020\end{array}$ & $\begin{array}{l}284 \text { with FIB } \\
\text { (64 vs 220) }\end{array}$ & 61.8 vs 43.9 & $\begin{array}{l}58.7 \pm 11 \text { vs } \\
46.1 \pm 14.1\end{array}$ & $p<0.001$ & $\begin{array}{l}\text { Severity } / 2 \text { based } \\
\text { on NHC China }\end{array}$ & $\begin{array}{l}4.5 \pm 1 \text { vs } \\
3.9 \pm 1.1\end{array}$ & $\mathrm{n} / \mathrm{a}$ & $\begin{array}{l}\text { On } \\
\text { admission }\end{array}$ & 27.6 vs 11.5 & 34.2 vs 12.6 & 17.1 vs 4.6 & Good \\
\hline
\end{tabular}


Table 1

Continued

\begin{tabular}{|c|c|c|c|c|c|c|c|c|c|c|c|c|c|c|c|c|c|}
\hline No & Author & $\begin{array}{l}\text { Study } \\
\text { design }\end{array}$ & Hospital & $\begin{array}{l}\text { Town, } \\
\text { Country }\end{array}$ & Period & Samples (n) & Male (\%) & Age, $y$ & $\begin{array}{l}\text { Age } \\
\text { difference }\end{array}$ & $\begin{array}{l}\text { Outcome } / \text { No. of } \\
\text { group }\end{array}$ & $\begin{array}{l}\text { FIB }(g / L) \\
\text { Mean } \pm \text { SD }\end{array}$ & $\begin{array}{l}\text { FDP }(\mu \mathrm{g} / \mathrm{ml}) \\
\text { Mean } \pm \text { SD }\end{array}$ & $\begin{array}{l}\text { Time of } \\
\text { diagnosis }\end{array}$ & HTN $(\%)$ & CVD $(\%)$ & DM $(\%)$ & $\begin{array}{l}\text { Study } \\
\text { quality }\end{array}$ \\
\hline 9 & $\begin{array}{l}\text { Han H, } 2020 \\
{[10]}\end{array}$ & Pros. & $\begin{array}{l}\text { Renmin Hospital } \\
\text { of Wuhan } \\
\text { University }\end{array}$ & $\begin{array}{l}\text { Wuhan, } \\
\text { China }\end{array}$ & $\begin{array}{l}\text { Jan 11-Feb } \\
10,2020\end{array}$ & 94 (45 vs 49) & $\mathrm{n} / \mathrm{a}$ & $\mathrm{n} / \mathrm{a}$ & n/a & $\begin{array}{l}\text { Severity//3 based } \\
\text { on NHC China }\end{array}$ & $\begin{array}{l}.94 \pm 1.87 \\
\text { vs } 5.1 \pm 1.16\end{array}$ & $\begin{array}{l}62.04 \pm \\
112.28 \text { vs } \\
7.92 \pm 11.38\end{array}$ & $\mathrm{n} / \mathrm{a}$ & $\mathrm{n} / \mathrm{a}$ & $\mathrm{n} / \mathrm{a}$ & $\mathrm{n} / \mathrm{a}$ & Fair \\
\hline 10 & $\begin{array}{l}\text { Han Y, } 2020 \\
{[23]}\end{array}$ & Retros. & $\begin{array}{l}\text { Renmin Hospital } \\
\text { of Wuhan } \\
\text { University }\end{array}$ & $\begin{array}{l}\text { Wuhan, } \\
\text { China }\end{array}$ & $\begin{array}{l}\text { Feb 1-18, } \\
2020\end{array}$ & 47 (24 vs 23 ) & $\begin{array}{l}70.83 \text { vs } \\
39.13\end{array}$ & $\begin{array}{l}65.08 \\
(31-87) \text { vs } \\
64.74 \\
(41-81)\end{array}$ & $p=0.926$ & $\begin{array}{l}\text { Severity/2 based } \\
\text { on ATS }\end{array}$ & $\begin{array}{l}5.19 \pm 0.41 \\
\text { vs } \\
4.43 \pm 0.67\end{array}$ & $\mathrm{n} / \mathrm{a}$ & $\begin{array}{l}\text { On } \\
\text { admission }\end{array}$ & $\begin{array}{l}41.67 \mathrm{vs} \\
34.78\end{array}$ & 16.67 vs 4.35 & $\begin{array}{l}16.67 \text { vs } \\
13.04\end{array}$ & Good \\
\hline 11 & $\begin{array}{l}\text { Shi P, } 2020 \\
{[19]}\end{array}$ & Retros. & $\begin{array}{l}\text { Xi'an, Ankang, } \\
\text { Baoji, Hanzhong, } \\
\text { Weinan, Xianyang, } \\
\text { Shangluo, Yan'an, } \\
\text { Tongchuan } \\
\text { Hospital }\end{array}$ & $\begin{array}{l}\text { Shaanxi, } \\
\text { China }\end{array}$ & $\begin{array}{l}\text { Jan } \\
23-\text { March } 7, \\
2020\end{array}$ & $\begin{array}{l}134 \text { (46 vs } \\
88)\end{array}$ & 54.3 vs 45.5 & $\begin{array}{l}56(46-66) \text { vs } \\
41(29-50)\end{array}$ & $p<0.000$ & $\begin{array}{l}\text { Severity/2 based } \\
\text { on NHC China }\end{array}$ & $\begin{array}{l}3.5 \pm 0.38 \text { vs } \\
3.15 \pm 0.31\end{array}$ & $\mathrm{n} / \mathrm{a}$ & $\begin{array}{l}\text { On } \\
\text { admission }\end{array}$ & 21.7 vs 11.4 & 8.7 vs 2.3 & 8.7 vs 5.7 & Good \\
\hline 12 & $\begin{array}{l}\text { Gao Y, } 2020 \\
{[16]}\end{array}$ & Retros. & $\begin{array}{l}\text { Fuyang Second } \\
\text { People's Hospital }\end{array}$ & $\begin{array}{l}\text { Fuyang, } \\
\text { China }\end{array}$ & $\begin{array}{l}\text { Jan } 23-\mathrm{Feb} 2 \\
2020\end{array}$ & 43 (15 vs 28$)$ & 60 vs 60.7 & $\begin{array}{l}45.20 \pm 7.68 \\
\text { vs } \\
42.96 \pm 14.00\end{array}$ & $p=0.503$ & Severity/2 & $\begin{array}{l}3.84 \pm 1.00 \\
\text { vs } \\
3.11 \pm 0.83\end{array}$ & n/a & $\mathrm{n} / \mathrm{a}$ & $\begin{array}{l}40.00 \text { vs } \\
25.00\end{array}$ & 6.67 vs 8.00 & 40.00 vs 3.57 & Good \\
\hline 13 & $\begin{array}{l}\text { Tang N, } \\
2020[2]\end{array}$ & Retros. & Tongji Hospital & $\begin{array}{l}\text { Wuhan, } \\
\text { China }\end{array}$ & $\begin{array}{l}\text { Jan 1-Feb } 3 \\
2020\end{array}$ & $\begin{array}{l}183 \text { (21 vs } \\
162)\end{array}$ & $\begin{array}{l}76.19 \text { vs } \\
50.61\end{array}$ & $\begin{array}{l}64.0 \pm 20.7 \\
\text { vs } \\
52.4 \pm 15.6\end{array}$ & $p<0.001$ & Mortality/2 & $\begin{array}{l}4.94 \pm 0.36 \\
\text { vs } \\
4.44 \pm 0.27\end{array}$ & $\begin{array}{l}10.65 \pm 3.59 \\
\text { vs } \\
4.08 \pm 0.06\end{array}$ & $\begin{array}{l}\text { On } \\
\text { admission }\end{array}$ & $\mathrm{n} / \mathrm{a}$ & $\mathrm{n} / \mathrm{a}$ & $\mathrm{n} / \mathrm{a}$ & Good \\
\hline 14 & $\begin{array}{l}\text { Zhang F, } \\
2020[24]\end{array}$ & Retros. & $\begin{array}{l}\text { Wuhan No.1 } \\
\text { Hospital }\end{array}$ & $\begin{array}{l}\text { Wuhan, } \\
\text { China }\end{array}$ & $\begin{array}{l}\text { Dec } 25 \\
2019-F e b 15 \\
2020\end{array}$ & 48 (17 vs 31$)$ & 70.6 vs 67.7 & $\begin{array}{l}78.65 \pm 8.31 \\
\text { vs } \\
66.16 \pm 13.66\end{array}$ & $p=0.001$ & Mortality/2 & $\begin{array}{l}4.40 \pm 1.48 \\
\text { vs } \\
4.66 \pm 1.49\end{array}$ & $\mathrm{n} / \mathrm{a}$ & $\begin{array}{l}\text { On } \\
\text { admission }\end{array}$ & 70.6 vs 64.5 & 23.5 vs 29.0 & 29.4 vs 16.1 & Good \\
\hline 15 & $\begin{array}{l}\mathrm{Hu} \text { C, } 2020 \\
{[25]}\end{array}$ & Retros. & $\begin{array}{l}\text { Sino-French New } \\
\text { City Branch of } \\
\text { Tongji Hospital }\end{array}$ & $\begin{array}{l}\text { Wuhan, } \\
\text { China }\end{array}$ & $\begin{array}{l}28 \text { Jan } \\
2020-11 \\
\text { March } 2020\end{array}$ & $\begin{array}{l}183(68 \text { vs } \\
115)\end{array}$ & $\begin{array}{l}73.53 \text { vs } \\
49.57\end{array}$ & $\begin{array}{l}68.44 \pm 9.94 \\
\text { vs } \\
60.54 \pm 13.19\end{array}$ & $p<0.001$ & Mortality/2 & $\begin{array}{l}5.38 \pm 2.04 \\
\text { vs } \\
4.50 \pm 1.44\end{array}$ & n/a & $\begin{array}{l}\text { On } \\
\text { admission }\end{array}$ & $\begin{array}{l}44.12 \text { vs } \\
37.39\end{array}$ & $\mathrm{n} / \mathrm{a}$ & $\begin{array}{l}20.59 \text { vs } \\
18.26\end{array}$ & Good \\
\hline 16 & $\begin{array}{l}\text { Yan Y, } 2020 \\
{[17]}\end{array}$ & Retros. & Tongji Hospital & $\begin{array}{l}\text { Wuhan, } \\
\text { China }\end{array}$ & $\begin{array}{l}10 \mathrm{Jan} \\
2020-24 \mathrm{Feb} \\
2020\end{array}$ & 48 (39 vs 9 ) & 76.9 vs 33.3 & $\begin{array}{l}70.5 \pm 10 \text { vs } \\
64.7 \pm 7.3\end{array}$ & $p=0.112$ & Mortality/2 & $\begin{array}{l}5.08 \pm 2.50 \\
\text { vs } \\
4.80 \pm 1.38\end{array}$ & $\mathrm{n} / \mathrm{a}$ & $\begin{array}{l}\text { On } \\
\text { admission }\end{array}$ & 52.8 vs 18.8 & 25 vs 4.7 & 36.1 vs 10.6 & Good \\
\hline 17 & $\begin{array}{l}\text { Zheng Y, } \\
2020[18]\end{array}$ & Retros. & $\begin{array}{l}\text { Chengdu Public } \\
\text { Health Clinical } \\
\text { Medical Center }\end{array}$ & $\begin{array}{l}\text { Chengdu, } \\
\text { China }\end{array}$ & $\begin{array}{l}16 \mathrm{Jan} \\
2020-20 \mathrm{Feb} \\
2020\end{array}$ & 99 (31 vs 68$)$ & $\begin{array}{l}19.19 \text { vs } \\
32.32\end{array}$ & $\begin{array}{l}63.8 \pm 16.51 \\
\text { vs } \\
42.5 \pm 15.51\end{array}$ & $p<0.001$ & Critically ill/2 & $\mathrm{n} / \mathrm{a}$ & $\begin{array}{l}6.72 \pm 8.36 \\
\text { vs } \\
2.65 \pm 2.33\end{array}$ & $\begin{array}{l}\text { On } \\
\text { admission }\end{array}$ & $\mathrm{n} / \mathrm{a}$ & $\mathrm{n} / \mathrm{a}$ & $\mathrm{n} / \mathrm{a}$ & Fair \\
\hline
\end{tabular}




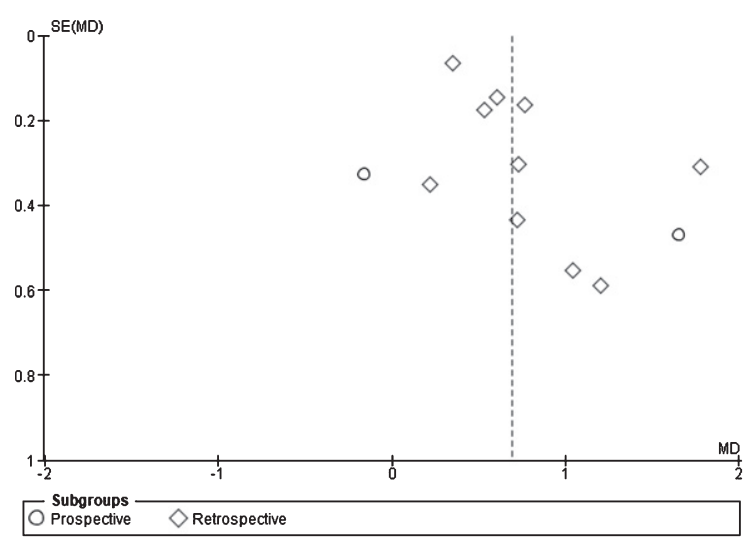

(A)

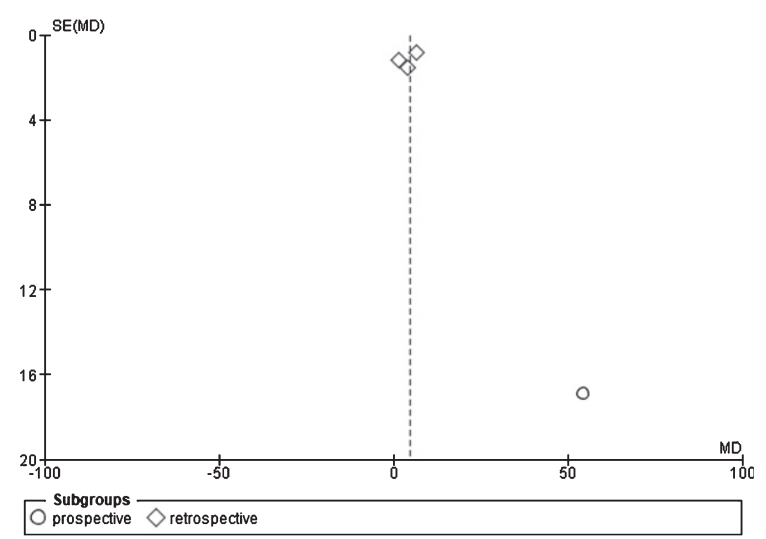

(C)

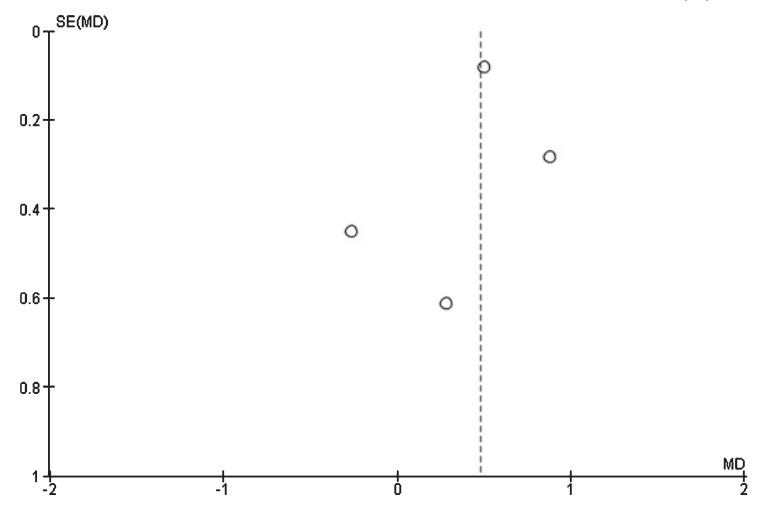

(B)

Fig. 2. Funnel plot shows no publication bias for (A) fibrinogen and severity $[p=0.154]$, but there are publication biases for (B) fibrinogen and mortality, and (C) fibrin degradation product and composite poor outcomes in patients with COVID-19.

The Egger's test showed there was no publication bias for fibrinogen in severity outcome $(p=0.154)$. We did not conduct Egger's test for other outcomes because there were less than ten included studies. Figure 2 shows that all funnel plots showed an asymmetrical appearance indicating publication bias in fibrinogen for mortality outcome and FDP for composite poor outcomes.

Random-effects meta-analysis revealed a higher mean of fibrinogen level on admission in patients with severe case compared to those with non-severe case as shown in Fig. 3A (12 studies, MD =0.69, [95\% CI: 0.44 to 0.94$], p<0.05 ; \mathrm{I} 2=72 \%, p<0.05)$. Subgroup analysis based on study design showed no difference result in prospective design subgroup. Sensitivity analysis using leave-one-out method showed that removal of Shi et al. [19]. reduced the heterogeneity with similar result ( $\mathrm{MD}=0.75,[95 \%$ CI: 0.47 to 1.04$], p<0.05 ; \mathrm{I} 2=64 \%, p<0.05)$.

Non-survivor group had a pooled higher mean difference of fibrinogen level on admission than survivor group as shown in Fig. 3B (4 studies, $\mathrm{MD}=0.48$ [95\% CI: 0.13 to 0.83 ], $p<0.05$; I2 $=38 \%$, $p=0.18$ ). There was no substantial heterogeneity found across the studies. We did not perform subgroup analysis based on study design because all included studies were retrospective observational.

Higher FDP on admission was found in poor outcome (composite of severity, critically ill, and mortality) compared to good outcome as shown in Fig. 3C (4 studies, MD =4.84 [95\% CI: 0.75 to $8.93], p<0.05 ; \mathrm{I} 2=86 \%, p<0.05)$. Subgroup analysis of retrospective design showed similar results. Sensitivity analysis showed that removal of Zheng et al. [14]. reduced the heterogeneity with similar result $(\mathrm{MD}=6.49$, [95\% CI: 1.45 to 11.53$], p<0.05 ; \mathrm{I} 2=81 \%, p<0.05)$. 
A

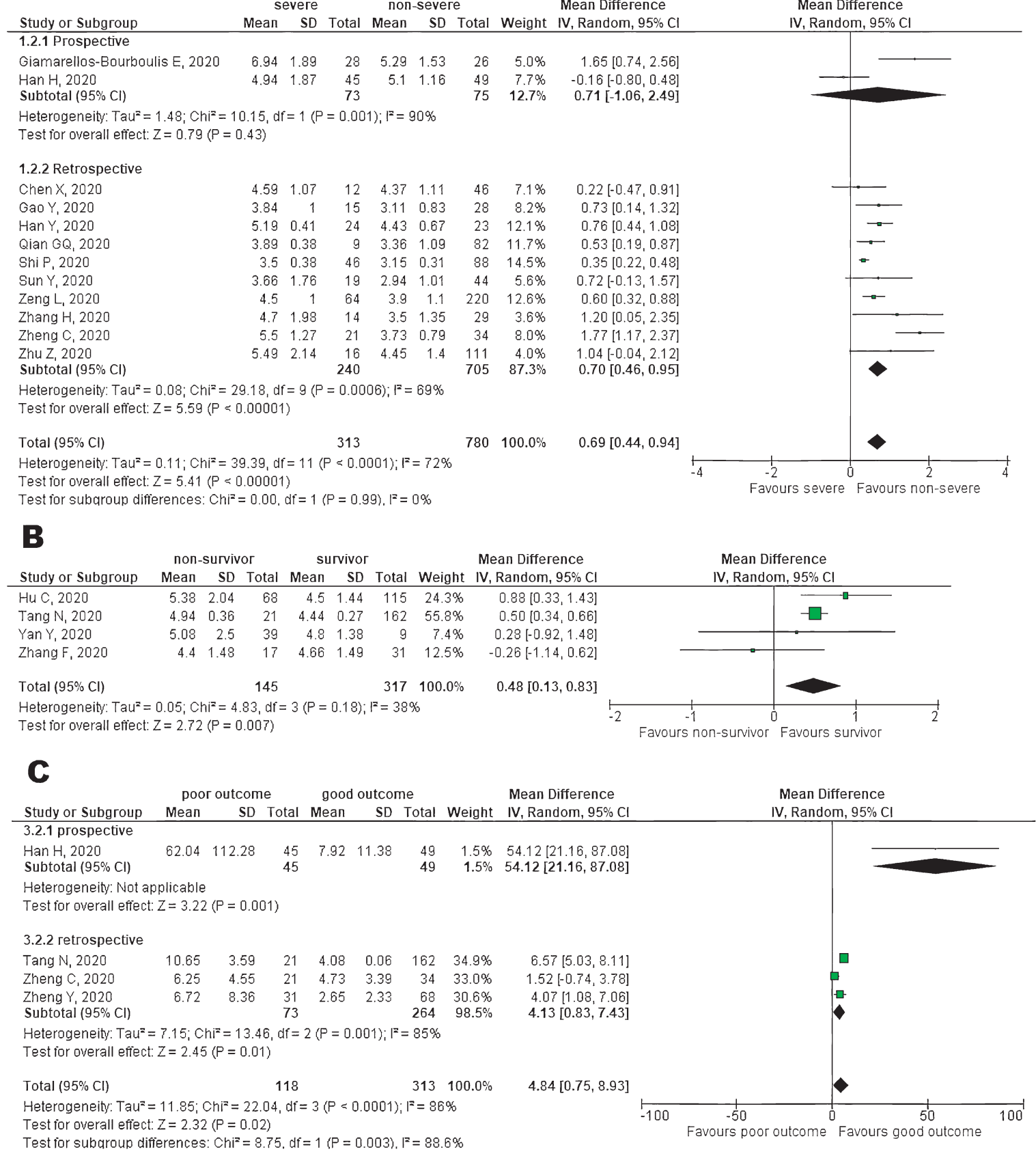

Fig. 3. (A) Forest plot of fibrinogen for severity; (B) Forest plot of fibrinogen for mortality; (C) Forest plot of fibrinogen degradation product for poor outcome.

\section{Discussion}

This meta-analysis showed that high fibrinogen levels on admission were significantly associated with severity and mortality. In addition, elevated FDP was significantly associated with composite poor outcome. Our results are in line with previous studies about the association between poor prognosis 
of COVID-19 and coagulation profile as appeared in early reports from China [3].

One study by Tang et al. reported that non-survivors had higher fibrinogen and FDP level than survivors on early stage of disease. In the late course of the disease, non-survivors had a significantly lower level of fibrinogen than survivors [2]. Another study reported that COVID-19 patient had high level of fibrinogen while DIC patient had low fibrinogen level [26].

The normal plasma concentration of fibrinogen is between 2 and $4 \mathrm{~g} / \mathrm{l}$; however, this concentration can rapidly increase under pathological conditions such as injury, infection, or inflammation [27]. Viral infections may induce severe complications such as acute respiratory distress syndrome and multiorgan failure which are often accompanied by hypercoagulation and DIC [28, 29]. The laboratory features of DIC vary widely depending on the stage encountered. In early DIC, there is compensated activation of the hemostatic system, however with progression to decompensated hemostatic activation, characteristic findings are observed. Fibrinogen levels vary; however, in advanced stages of DIC, fibrinogen levels decrease [26]. The exact mechanism for coagulopathy in COVID-19 is not yet identified. COVID-19 is able to directly attack vascular endothelial cells expressing high levels of ACE2, leading to abnormal coagulation and sepsis. The virus enters endothelial cells by endocytosis via the binding of its spike glycoprotein to a cellular receptor which facilitates viral attachment to the surface of target cells [30, 31].

The prevalence of VTE, particularly in critically ill/severe patients suffering from COVID-19, was reported as much as 25-27\% [32]. VTE is often associated with plasma hypercoagulability. An elevated plasma fibrinogen level significantly increases the risk of VTE. These findings suggest hyperfibrinogenemia is not merely a biomarker of thrombotic risk, but is causative in the etiology of venous thrombosis [33].

Hyperfibrinolysis was present in 97\% of COVID-19 patients [34]. Fibrin and FDP are formed during intravascular clotting [35]. A study observed an increase of activated plasmin and fibrinolytic activity in COVID-19 patients which resulted in the elevated FDP [36]. At late stages of COVID-19, FDP were reported to be moderately or markedly elevated in all COVID-19 deaths [37].

Lung computed tomography (CT) scan shows an important role in the detection of thromboembolic events in COVID-19. Abdominal microcirculatory disorders, as well as central or peripheral pulmonary embolism was also found in severe cases of COVID-19. As the disease worsened, the laboratory parameter of fibrinogen was increased to some varying degree, and multiple lesions in CT scan were progressively noticed $[38,39]$. Imaging modality which is greatly beneficial in detecting thrombotic events occurrence in these cases in addition to lung CT scan, is contrast-enhanced ultrasound (CEUS). Peripherally accentuated consolidation with irregular hyperemia was found in the CEUS and also in the CT examination in cases of pulmonary thrombus embolism [40]. CEUS can dynamically record the mesenteric arterial and venous blood flow, thus enabling us to quickly rule out abdominal infarction [41].

Our meta-analysis suggests that elevated fibrinogen on admission can also be a marker of poor prognosis in patients with COVID-19. During a pandemic, risk stratification in triage is necessary, and fibrinogen levels can be one of potential indicators of high-risk patients. While D-dimer level is often used to aid the decision of starting an anticoagulant, fibrinogen level might direct the physician to stop anticoagulation. It should be noted that, like all cases of DIC, patients may progress to a hypercoagulable state when fibrinogen levels begin to decrease. At this point, stopping anticoagulation should be considered.

To the best of authors knowledge, our meta-analysis comprising of 17 studies is the largest metaanalysis evaluating the prognostic role of fibrinogen on admission in COVID-19. However, several limitations should be noted in our study. First, there was substantial heterogeneity across studies. Most of included studies were retrospective with a relatively small sample size. Second, the presence of publication bias. The analysis of this study was performed during pandemic; many areas affected by 
COVID-19 have not published the data yet. Most of the studies included were from mainland China. The ethnic and geographical differences might distort the results.

\section{Conclusions}

Our meta-analysis demonstrated that elevated fibrinogen and fibrin degradation products on admission were associated with an increased risk of poor outcome in COVID-19 patients.

\section{Data availability}

The data supporting this meta-analysis are from previously reported studies and datasets, which have been cited.

\section{Conflict of interest}

The authors declare that the research was conducted in the absence of any commercial or financial relationships that could be considered as a potential conflict of interests.

\section{Acknowledgments}

This work did not receive specific funding, but was performed as part of Johanes Nugroho employment at the Department of Cardiology and Vascular Medicine, Universitas Airlangga/Dr. Soetomo General Hospital, East Java, Indonesia.

\section{References}

[1] Giannis D, Ziogas IA, Gianni P. Coagulation disorders in coronavirus infected patients: COVID-19, SARS- CoV-1, MERS-CoV and lessons from the past 2020. J Clin Virol. 2020;127:104362.

[2] Tang N, Li D, Wang X, Sun Z. Abnormal coagulation parameters are associated with poor prognosis in patients with novel coronavirus pneumonia. J Thromb Haemost. 2020;18:844-7.

[3] Sakka M, Connors JM, Hékimian G, Martin-Toutain I, Crichi B, Colmegna I, et al. Association between D-Dimer levels and mortality in patients with coronavirus disease 2019 (COVID-19): a systematic review and pooled analysis. JMV-Journal Médecine Vasc. 2020;2019:1-7.

[4] Chen G, Zhao J, Ning Q, Cao Y, Huang D, Wang H, et al. Clinical and immunological features of severe and moderate coronavirus disease 2019. J Clin Invest. 2020;130(5):2620-9.

[5] Hutton B, Salanti G, Caldwell DM, Chaimani A, Schmid CH, Cameron C, et al. The PRISMA extension statement for reporting of systematic reviews incorporating network meta-analyses of health care interventions: Checklist and explanations. Ann Intern Med 2015;162:777-84

[6] Higgins J, Thomas J, Chandler J, Cumpston M, Li T, Page M, et al. Cochrane Handbook for Systematic Reviews of Interventions version 6.0 (updated July 2019). Cochrane 2019. https://training.cochrane.org/handbook. (accessed May 20, 2020).

[7] National Institute of Health. Study Quality Assessment Tools | National Heart, Lung, and Blood Institute (NHLBI) n.d. https://www.nhlbi.nih.gov/health-topics/study-quality-assessment-tools. (accessed May 20, 2020).

[8] Egger M, Smith GD, Schneider M, Minder C. Bias in meta-analysis detected by a simple, graphical test measures of funnel plot asymmetry. Bmj. 1997;315:629-34

[9] Wan X, Wang W, Liu J, Tong T. Estimating the sample mean and standard deviation from the sample size, median, range and/or interquartile range. BMC Med Res Methodol. 2014;14:1-13. 
[10] Han H, Yang L, Liu R, Liu F, Wu KL, Li J, et al. Prominent changes in blood coagulation of patients with SARS-CoV-2 infection. Clin Chem Lab Med. 2020;58:1116-20.

[11] Qian G-Q, Yang N-B, Ding F, Ma AHY, Wang Z, Shen Y, et al. Epidemiologic and clinical characteristics of 91 hospitalized patients with COVID-19 in Zhejiang, China: A retrospective, multi-centre case series [published online ahead of print, 2020 Mar 17]. QJM. 2020;hcaa089. doi:10.1093/qjmed/hcaa089

[12] Zhu Z, Cai T, Fan L, Lou K, Hua X, Huang Z, et al. Clinical value of immune-inflammatory parameters to assess the severity of coronavirus disease 2019. Int J Infect Dis. 2020;95:332-9.

[13] Giamarellos-Bourboulis EJ, Netea MG, Rovina N, Akinosoglou K, Antoniadou A, Antonakos N, et al. Complex immune dysregulation in COVID-19 patients with severe respiratory failure. Cell Host Microbe. 2020;27:992-1000.e3.

[14] Zheng C, Wang J, Guo H, Lu Z, Ma Y, Zhu Y, et al. Risk-adapted treatment strategy for COVID-19 patients. Int J Infect Dis. 2020;94:74-7.

[15] Sun Y, Dong Y, Wang L, Xie H, Li B, Chang C, et al. Characteristics and prognostic factors of disease severity in patients with COVID-19: The Beijing experience. J Autoimmun. 2020:102473. https://doi.org/10.1016/j.jaut.2020.102473.

[16] Gao Y, Li T, Han M, Li X, Wu D, Xu Y, et al. Diagnostic utility of clinical laboratory data determinations for patients with the severe COVID-19. J Med Virol. 2020;92:791-6.

[17] Yan Y, Yang Y, Wang F, Ren H, Zhang S, Shi X, et al. Clinical characteristics and outcomes of patients with severe covid-19 with diabetes. BMJ Open Diabetes Res Care. 2020;8:e001343.

[18] Zheng Y, Xu H, Yang M, Zeng Y, Chen H, Liu R, et al. Epidemiological characteristics and clinical features of 32 critical and 67 noncritical cases of COVID-19 in Chengdu. J Clin Virol. 2020;127:104366.

[19] Shi P, Ren G, Yang J, Li Z, Deng S, Li M, et al. Clinical characteristics of imported and second-generation COVID-19 cases outside Wuhan, China: A multicenter retrospective study. medRxiv. 2020;2020.04.19.20071472. doi:10.1101/2020.04.19.20071472.

[20] Zhang H, Wang X, Fu Z, Luo M, Zhang Z, Zhang K, et al. Potential factors for prediction of disease severity of COVID-19 patients. medRxiv. 2020;2020.03.20.20039818. doi:10.1101/2020.03.20.20039818.

[21] Chen X, Zheng F, Qing Y, Ding S, Yang D, Lei C, et al. Epidemiological and clinical features of 291 cases with coronavirus disease 2019 in areas adjacent to Hubei, China: a double-center observational study. medRxiv. 2020;2020.03.03.20030353. doi:10.1101/2020.03.03.20030353

[22] Zeng L, Li J, Liao M, Hua R, Huang P, Zhang M, et al. Risk assessment of progression to severe conditions for patients with COVID-19 pneumonia: a single-center retrospective study. medRxiv. 2020;2020.03.25.20043166. doi:10.1101/2020.03.25.20043166

[23] Han Y, Zhang H, Mu S, Wei W, Jin C, Xue Y, et al. Lactate dehydrogenase, a Risk Factor of Severe COVID-19 Patients. medRxiv. 2020;2020.03.24.20040162. doi:10.1101/2020.03.24.20040162

[24] Zhang F, Yang D, Li J, Gao P, Chen T, Cheng Z, et al. Myocardial injury is associated with in-hospital mortality of confirmed or suspected COVID-19 in Wuhan, China: A single center retrospective cohort study. medRxiv. 2020;2020.03.21.20040121. doi:10.1101/2020.03.21.20040121

[25] Hu C, Liu Z, Jiang Y, Zhang X, Shi O, Xu K, et al. Early prediction of mortality risk among severe COVID-19 patients using machine learning. medRxiv. 2020:2020.04.13.20064329. doi:10.1101/2020.04.13.20064329

[26] Becker RC. COVID-19 update: Covid-19-associated coagulopathy. J Thromb Thrombolysis. 2020;50(1):54-67.

[27] Göbel K, Eichler S, Wiendl H, Chavakis T, Kleinschnitz C, Meuth SG. The coagulation factors fibrinogen, thrombin, and factor XII in inflammatory disorders-a systematic review. Front Immunol. 2018;9:1731.

[28] Di Micco P, Russo V, Carannante N, Imparato M, Rodolfi S, Cardillo G, et al. Clotting factors in COVID-19: Epidemiological association and prognostic values in different clinical presentations in an Italian cohort. J Clin Med. 2020;9:1371.

[29] Sardu C, Gambardella J, Morelli MB, Wang X, Marfella R, Santulli G. Is COVID-19 an endothelial disease? clinical and basic evidence. Clin Basic Evid. 2020;9:1-26.

[30] Levy M, van der Poll T. Coagulation and sepsis. Thromb Res. 2017;149:38-44.

[31] Jung F, Krüger-Genge A, Franke RP, Hufert F, Küpper JH. COVID-19 and the endothelium. Clin Hemorheol Microcirc. 2020;75:7-11.

[32] Klok FA, Kruip MJHA, van der Meer NJM, Arbous MS, Gommers DAMPJ, Kant KM, et al. Incidence of thrombotic complications in critically ill ICU patients with COVID-19. Thromb Res. 2020;191:145-7.

[33] Machlus KR, Cardenas JC, Church FC, Wolberg AS. Causal relationship between hyperfibrinogenemia, thrombosis, and resistance to thrombolysis in mice. Blood. 2011;117(18):4953-63

[34] Zhang B, Zhou X, Qiu Y, Song Y, Feng F, Feng J, et al. Clinical characteristics of 82 cases of death from COVID-19. PLoS ONE. 2020;15(7):e0235458

[35] Hemker HC, Fekkes N, Hensen A, Schrijver H, Loeliger EA. Quantitation of circulating fibrinogen breakdown products in intravascular clotting. Thromb Diath Haemorrh Suppl. 1966;20:227-34. 
[36] Ji H-L, Zhao R, Matalon S, Matthay MA. Elevated plasmin(ogen) as a common risk factor for COVID-19 susceptibility. Physiol Rev. 2020;100(3):1065-75.

[37] Ponti G, Maccaferri M, Ruini C, Tomasi A, Ozben T. Biomarkers associated with COVID-19 disease progression. Crit Rev Clin Lab Sci. 2020;0(0):1-11.

[38] Jiang X, Yin Z, Wang T, Zhai N, Lu F, Zhan C, et al. Covid-19 dynamic computed tomography (CT) performance and observation of some laboratory indicators. Med Sci Monit. 2020;26:1-7

[39] Li M, Lei P, Zeng B, et al. Coronavirus Disease (COVID-19): Spectrum of CT Findings and Temporal Progression of the Disease. Academic Radiology. 2020;27:603-8

[40] Jung EM, Stroszczynski C, Jung F. Contrast enhanced ultrasound (CEUS) to assess pleural pulmonal changes in severe COVID-19 infection: First results. Clin Hemorheol Microcirc. 2020;75(1):19-26.

[41] Jung EM, Stroszczinski C, Jung F. Contrast enhanced ultrasonography (CEUS) to detect abdominal microcirculatory disorders in severe cases of COVID-19 infection: First experience. Clin Hemorheol Microcirc. 2020;74(4):353-61. 